ARTICLE

\title{
Evolution and expansion of Li concentration gradient during charge-discharge cycling
}

\author{
Byeong-Gyu Chae (10 ${ }^{1,3,4 凶}$, Seong Yong Park (10 1,3,4凶${ }^{\text {, Jay Hyok Song }}{ }^{2}$, Eunha Lee (D) ${ }^{1}$ \& Woo Sung Jeon ${ }^{1}$
}

To improve the performance of Li-ion batteries (LIBs), it is essential to understand the behaviour of Li ions during charge-discharge cycling. However, the analytical techniques for observing the $\mathrm{Li}$ ions are limited. Here, we present the complementary use of scanning transmission electron microscopy and atom probe tomography at identical locations to demonstrate that the evolution of the local Li composition and the corresponding structural changes at the atomic scale cause the capacity degradation of $\mathrm{Li}\left(\mathrm{Ni}_{0.80} \mathrm{Co}_{0.15} \mathrm{Mn}_{0.05}\right) \mathrm{O}_{2}$ (NCM), an LIB cathode. Using these two techniques, we show that a Li concentration gradient evolves during cycling, and the depth of the gradient expands proportionally with the number of cycles. We further suggest that the capacity to accommodate Li ions is determined by the degree of structural disordering. Our findings provide direct evidence of the behaviour of $\mathrm{Li}$ ions during cycling and thus the origin of the capacity decay in LIBs.

\footnotetext{
${ }^{1}$ Analytical Engineering Group, Material Research Center, Samsung Advanced Institute of Technology, Samsung Electronics Co., Ltd., Suwon, Republic of Korea. ${ }^{2}$ Materials Development Group 1, Samsung SDI, Suwon, Republic of Korea. ${ }^{3}$ These authors contributed equally: Byeong-Gyu Chae, Seong Yong Park.

${ }^{4}$ These authors jointly supervised this work: Byeong-Gyu Chae, Seong Yong Park. ${ }^{凶}$ email: bg.chae@samsung.com; sydra.park@samsung.com
} 
R echargeable Li-ion batteries (LIBs) have attracted great interest due to their explosive increase in demand for devices ranging from small portable electronics to large energy-storage devices ${ }^{1-8}$. This rapid expansion necessitates further improving their performance with regard to their capacity, charging speed, lifetime and safety. Many studies have investigated the action/degradation mechanisms of LIB cathode materials to understand the underlying physics in order to develop performance improvement strategies ${ }^{9-15}$. However, these mechanisms were primarily proposed based on the observation of the positions and chemical states of the transition metals (TMs) and oxygen atoms, but not those of $\mathrm{Li}$ ions, which are directly responsible for the battery operation because of the lack of reliable experimental techniques for the analysis of the Li-ion distribution ${ }^{9-16}$.

Among widely used experimental techniques, scanning transmission electron microscopy (STEM) and advanced X-ray techniques have provided invaluable information, such as the crystalline lattice structure ${ }^{17-20}$ and chemical sates ${ }^{21-30}$. Thus far, however, they lack the ability to directly quantify the charge carrier in LIBs, Li ions, at high spatial resolution. Therefore, it is still difficult to comparatively study and explain the capacity decay mechanism of LIBs using the aforementioned techniques and lifetime testing. To achieve this aim, we employed STEM, which reveals the atomic arrangements, together with atom probe tomography (APT) at the same location, which provides 3D quantitative information on the constituent elements, including $\mathrm{Li}$ ions, with a sensitivity of $\sim 10$ parts per million ${ }^{31-34}$.

We report the origin of the irreversible capacity loss of a $\mathrm{Li}\left(\mathrm{Ni}_{0.80} \mathrm{Co}_{0.15} \mathrm{Mn}_{0.05}\right) \mathrm{O}_{2}(\mathrm{NCM})$ cathode material subjected to numerous charge-discharge cycles by correlating its structural evolution with its quantitative $3 \mathrm{D}$ composition. We selected an NCM cathode with a high Ni content, which was recently commercialised owing to its high capacity and low material cost $t^{8,10,35}$. Its structural and corresponding compositional changes during charge-discharge cycling were investigated to further understand the capacity degradation mechanism. We further demonstrate that the $\mathrm{Li}$ concentration is proportional to the amount of $\mathrm{Li}$ accommodation sites, which in turn is determined by the structural evolution. Our findings provide a new pathway for addressing the capacity decay in LIBs by understanding the inherent behaviour of $\mathrm{Li}$ ions.

\section{Results}

Capacity fade and the direct observation of Li. To clarify the correlation between the capacity fade and the loss of $\mathrm{Li}$, we examine the change in the capacity fade with both the global and local $\mathrm{Li}$ losses during cycling. Figure 1a reveals that the capacity of the NCM-based full cell (Supplementary Fig. 1) fades continuously to $137.5 \mathrm{mAh} \mathrm{g}^{-1}$, which is $76.0 \%$ of the initial specific capacity, after 300 charge-discharge cycles at a C-rate of 1 C. Supplementary a

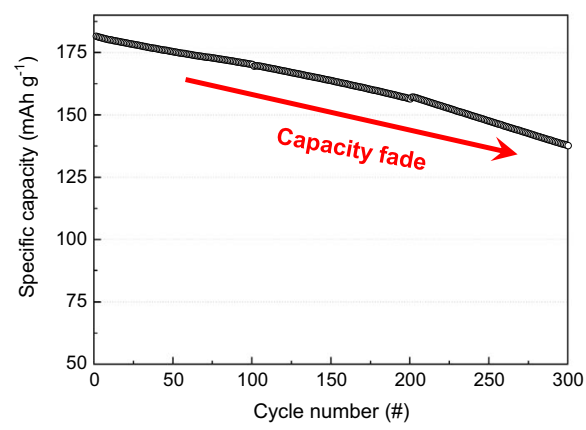

b

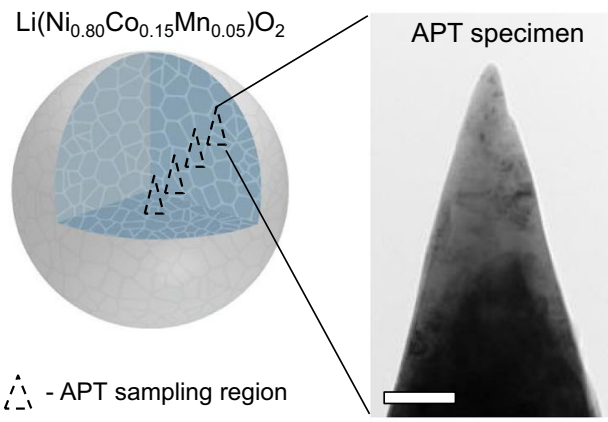

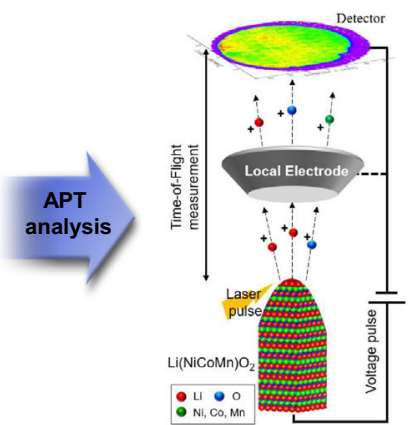

Surface Center Surface

d

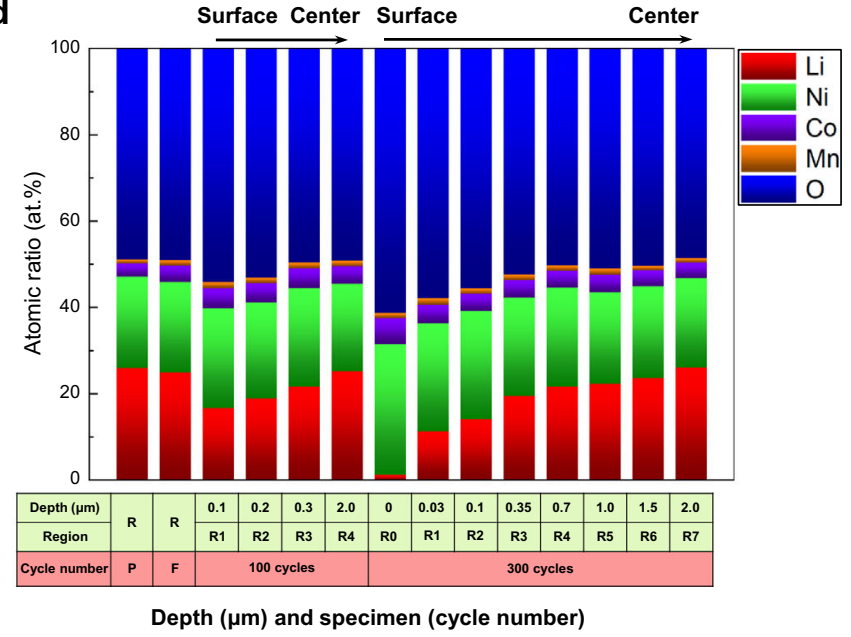

Fig. 1 Evolution of Li concentration gradient in NCM particle after charge-discharge cycles. a Specific capacity as a function of the number of cycles, revealing capacity fading in the NCM cell under a C-rate of $1 \mathrm{C}$ at $45^{\circ} \mathrm{C}$. $\mathbf{b}$ Schematic illustrations of site-specific APT and STEM analysis along the depth of secondary particles to correlate structural degradation with quantitative Li concentration. Scale bar is $200 \mathrm{~nm}$. c, d Comparison of the normalised atomic ratio of $\mathrm{Li}(\mathbf{c})$ and compositional changes (d), as measured by APT, along the depth of the particles before and after cycling. Pristine and formed NCM are denoted by $\mathrm{P}$ and $\mathrm{F}$, respectively. The Li concentration gradient begins to evolve after cycling, and the depth of this gradient expands as the number of cycles increases. Error bars for each data points are from the standard deviation of measured ratios from multiple samples. 


\begin{tabular}{|c|c|c|c|c|}
\hline \multirow[t]{2}{*}{ Specimen } & \multicolumn{4}{|c|}{ Atomic concentration (weight \%) } \\
\hline & Li & $\mathbf{N i}$ & Co & $\mathbf{M n}$ \\
\hline $\begin{array}{l}\text { NCM-pristine } \\
\text { NCM-100 cycles } \\
\text { NCM-300 cycles }\end{array}$ & $\begin{array}{l}1.04(6.77) \\
0.94(6.16) \\
0.83(5.44)\end{array}$ & $\begin{array}{l}0.80(44.31) \\
0.80(44.36) \\
0.80(44.58)\end{array}$ & $\begin{array}{l}0.15(8.24) \\
0.15(8.23) \\
0.15(8.29)\end{array}$ & $\begin{array}{l}0.05(2.46) \\
0.05(2.46) \\
0.05(2.48)\end{array}$ \\
\hline
\end{tabular}

Table 2 Site-specific quantitative APT results along the depth of pristine $\mathrm{Li}\left(\mathrm{Ni}_{0.80} \mathrm{CO}_{0.15} \mathrm{Mn}_{0.05}\right) \mathrm{O}_{2}$ (NCM) and NCM subjected to the formation, 100 cycles and 300 cycles (the atomic ratio is normalised to obtain a total metal fraction of 1).

\begin{tabular}{|c|c|c|c|c|c|c|}
\hline \multicolumn{2}{|l|}{ Specimen } & \multicolumn{5}{|c|}{ Atomic ratio } \\
\hline Process & $\begin{array}{l}\text { Region (depth from } \\
\text { the surface) }\end{array}$ & Li & $\mathbf{N i}$ & Co & Mn & 0 \\
\hline NCM-pristine & $\begin{array}{l}R \text { (from surface to } \\
\text { centre) }\end{array}$ & 1.03 & 0.84 & 0.12 & 0.04 & 1.94 \\
\hline NCM formation & $\begin{array}{l}\mathrm{R} \text { (from surface to } \\
\text { centre) }\end{array}$ & 0.96 & 0.81 & 0.15 & 0.05 & 1.89 \\
\hline \multirow[t]{4}{*}{ NCM-100 cycles } & $\mathrm{R} 1(<100 \mathrm{~nm})$ & 0.57 & 0.79 & 0.16 & 0.05 & 1.85 \\
\hline & $\mathrm{R} 2(\sim 200 \mathrm{~nm})$ & 0.68 & 0.79 & 0.16 & 0.05 & 1.90 \\
\hline & R3 $(\sim 300 \mathrm{~nm})$ & 0.76 & 0.79 & 0.16 & 0.05 & 1.73 \\
\hline & $\mathrm{R} 4(\sim 2 \mu \mathrm{m})$ & 0.99 & 0.79 & 0.16 & 0.05 & 1.92 \\
\hline NCM- & RO (surface) & 0.03 & 0.81 & 0.16 & 0.03 & 1.63 \\
\hline \multirow[t]{7}{*}{300 cycles } & $\mathrm{R} 1(<30 \mathrm{~nm})$ & 0.37 & 0.81 & 0.14 & 0.05 & 1.88 \\
\hline & $\mathrm{R} 2(\sim 100 \mathrm{~nm})$ & 0.47 & 0.83 & 0.13 & 0.04 & 1.84 \\
\hline & R3 $(\sim 350 \mathrm{~nm})$ & 0.69 & 0.81 & 0.14 & 0.05 & 1.86 \\
\hline & $\mathrm{R} 4(\sim 700 \mathrm{~nm})$ & 0.77 & 0.82 & 0.14 & 0.04 & 1.79 \\
\hline & R5 $(\sim 1.0 \mu \mathrm{m})$ & 0.84 & 0.80 & 0.15 & 0.05 & 1.91 \\
\hline & $\mathrm{R} 6(\sim 1.5 \mu \mathrm{m})$ & 0.91 & 0.82 & 0.14 & 0.04 & 1.94 \\
\hline & $\mathrm{R} 7(\sim 2.5 \mu \mathrm{m})$ & 1.03 & 0.82 & 0.14 & 0.04 & 1.91 \\
\hline
\end{tabular}

Fig. 2 shows the charge-discharge curves of the cell cycled in the voltage range of $2.8-4.35 \mathrm{~V}$ at $0.2 \mathrm{C}$ and $45^{\circ} \mathrm{C}$ as a formation process (hereafter, the corresponding cell is referred to as NCM formation). In addition, inductively coupled plasma-atomic emission spectroscopy (ICP-AES) confirms a significant loss of Li from the cathode after battery cycling (Table 1). The atomic ratio of asprepared NCM (hereafter referred to as NCM-pristine) is Li:Ni:Co: $\mathrm{Mn}=1.04: 0.80: 0.15: 0.05$, in agreement with the intended stoichiometry. However, the Li concentration decreased dramatically with the number of cycles, whereas the composition of the TMs remained almost the same, as shown in Table 1. The atomic ratio of $\mathrm{Li}$ in NCM decreased continuously from 1.04 in NCM-pristine to 0.94 and 0.83 after 100 and 300 cycles of device operation, respectively (the cells subjected to 100 and 300 cycles are hereafter referred to as NCM-100 cycles and NCM-300 cycles, respectively). These results suggest a direct correlation between the amount of $\mathrm{Li}$ loss and the corresponding capacity degradation of the battery. However, both the distribution and local quantitative information of $\mathrm{Li}$ ions remain unknown.

With this combined approach, we directly observed the Li ions in $3 \mathrm{D}$ and quantified the Li loss (Fig. 1b). NCM particles are spherical with diameters of $\sim 10 \mu \mathrm{m}$, and each NCM particle consists of hundreds of smaller $(200-600 \mathrm{~nm})$ primary particles (Supplementary Figs. 3 and 4). We quantitatively analysed the compositions of NCM-pristine, NCM formation, NCM-100 cycles and NCM-300 cycles as a function of the radial direction from the surface of a secondary particle in contact with the liquid electrolyte, which acts as a pathway for the extraction and insertion of Li ions (Table 2, Fig. 1b-d and Supplementary Fig. 4). Notably, the Li insertion/extraction reaction is active at the particle surface, so the location, quantity and uniformity of the $\mathrm{Li}$ remaining in the cathode should be clarified.

In the case of compound materials, however, the accuracy of APT's compositional measurements depends on the analysis conditions such as the laser pulse energy ${ }^{36-41}$. We thus optimised these conditions to accurately quantify the composition without an under/overestimation. Under these conditions, the APT of NCM-pristine revealed the ideal stoichiometry when we applied a laser pulse energy of $25 \mathrm{pJ}$ at $30 \mathrm{~K}$. Its atomic ratio as determined by APT was Li:Ni:Co:Mn:O = 1.03:0.84:0.12:0.04:1.94 (Table 2 and Supplementary Fig. 5), in good agreement with the values from ICP-AES (Table 1). We also used APT to determine the atomic ratio of NCM formation, which was Li:Ni:Co:Mn: $\mathrm{O}=0.96: 0.81: 0.15: 0.05: 1.89$ (Table 2), almost the same as that of NCM-pristine. However, the cycled NCM showed a substantial Li deficiency, with an atomic fraction of Li less than 1.00, except for the most central regions of the particle. Further, the Li content tended to decrease with the increasing number of cycles, similar to the findings from ICP-AES. The APT results hence confirm that the Li content dramatically changed with the depth below the particle surface as well as the number of cycles.

Evolution/expansion of $\mathrm{Li}$ concentration gradient. The Li distribution in NCM subjected to repeated charge-discharge cycles was remarkably different from those in NCM-pristine and NCM formation (Fig. 1c, d and Supplementary Fig. 6). The APT result of NCM-100 cycles showed a severe Li deficiency at a distance of less than $100 \mathrm{~nm}$ from the top surface of the sample (100 cycles-Region 1; for simplicity, the different regions are hereafter referred to as R1, R2, etc., as defined in Table 2). In this region, the atomic ratio determined by APT is Li:Ni:Co:Mn: $\mathrm{O}=0.57: 0.79: 0.16: 0.05: 1.85$, indicating a deviation from those of NCM-pristine and NCM formation (Table 2). Specifically, the atomic fraction of Li decreased significantly from 1.03 for NCMpristine and 0.96 for NCM formation to 0.57 for R1 in NCM-100 cycles. A Li deficiency was still observed $\sim 200$ and $\sim 300 \mathrm{~nm}$ below the top surface (100 cycles-R2 and R3, respectively), but it was less pronounced than that of 100 cycles-R1 (Table 2). In contrast, $\sim 2 \mu \mathrm{m}$ below the surface, the composition was almost the same as that of the pristine sample (Table 2). The results of the quantitative APT analysis thus prove that a significant Li loss occurs at the top surface and that the amount of Li increases with increasing depth from the top surface of the particle, which can be described as a $\mathrm{Li}$ concentration gradient along the radial direction of the particle after 100 cycles.

The APT analysis of NCM-300 cycles also demonstrates the evolution of a Li concentration gradient along the radial direction and further reveals that this gradient region expanded. The atomic fraction of $\mathrm{Li}$ at the top surface (R0) and at depths of $\sim 30 \mathrm{~nm}$ (R1), $100 \mathrm{~nm}$ (R2), 350 nm (R3), 700 nm (R4), 1 $\mu \mathrm{m}$ (R5), $\sim 1.5 \mu \mathrm{m}$ (R6) and $\sim 2.5 \mu \mathrm{m}$ (R7) below the top surface are $0.03,0.37,0.47,0.69,0.77,0.84,0.91,1.03$, respectively. Thus, as clearly shown in Fig. 1c and Table 2, the amount of Li gradually increased from the surface to the centre of the particles in both NCM-300 cycles and NCM-100 cycles. However, in NCM-300 cycles, the Li-depleted region extended beyond $\sim 1.5 \mu \mathrm{m}$ below the surface. In addition, at a similar depth, the amount of Li in NCM300 cycles is noticeably lower than that in NCM-100 cycles, revealing that the $\mathrm{Li}$ concentration gradient extends deeper in NCM-300 cycles owing to an increase in the Li-depleted area. Thus, we suggest that the $\mathrm{Li}$ concentration gradient evolves during cycling, and the gradient region increases proportionally to the number of cycles. 
a

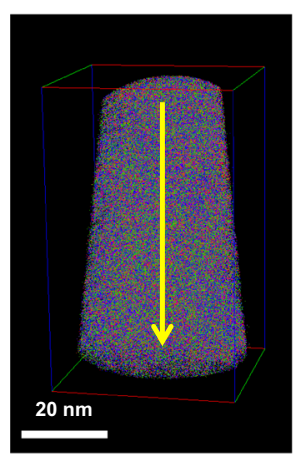

C

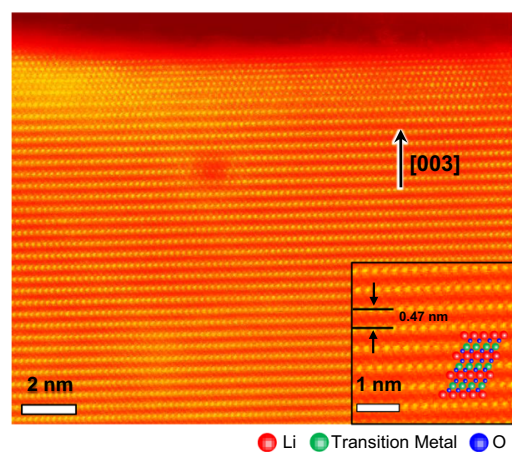

b

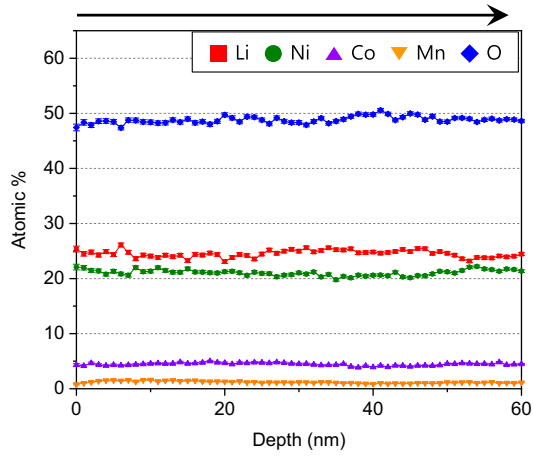

d

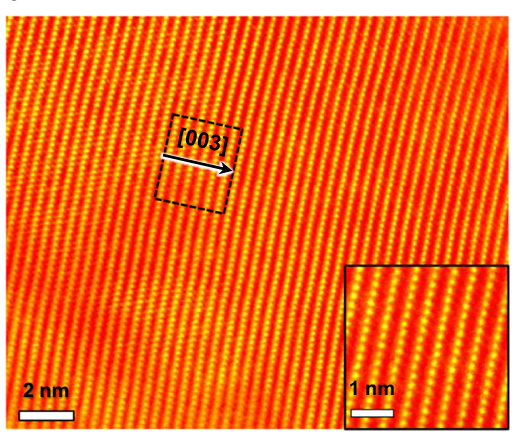

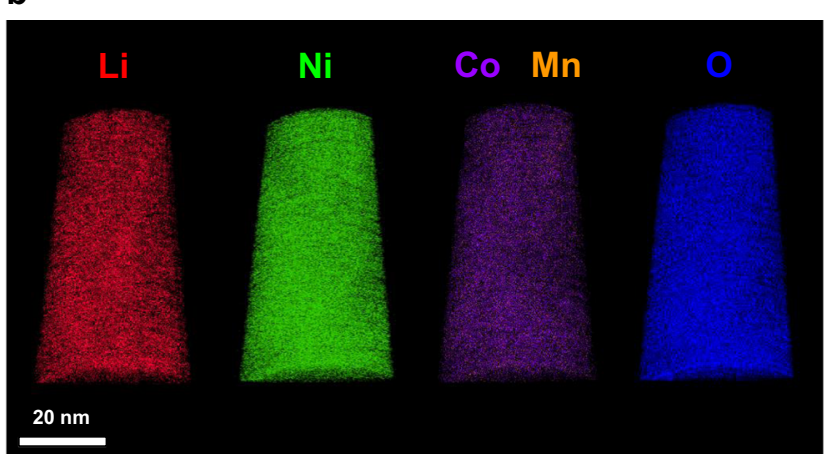

e

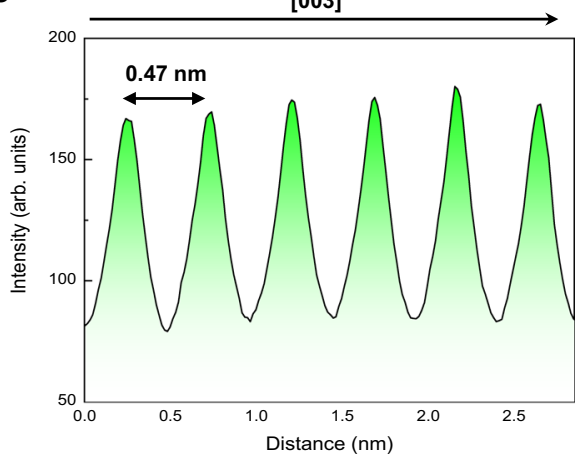

Fig. 2 Homogenous distribution of Li and the layered structure in NCM-pristine. a APT depth profile showing a homogeneous distribution of the

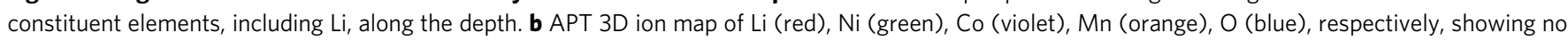
elemental segregation. Error bars show the standard deviation of each dataset. c, d STEM-HAADF images along [110] zone axis. A layered structure is clearly observed both at the surface (c) and in the interior (inset: magnified image) (d). e HAADF intensity profile along the region enclosed by the dotted square in (d), which shows the absence of TM migration.

Structural disordering and $\mathrm{Li}$ concentration. To reveal the origin of the $\mathrm{Li}$ concentration gradient, we investigated the microstructural changes associated with the evolution of this gradient by using site-specific STEM and APT analyses. The APT measurements near the surface of NCM-pristine provided atomic ratios that were in good agreement with the data determined by ICP-AES (Fig. 2a). The trends in the Li loss during cycling in the APT and ICP-AES results are also similar, showing a greater $\mathrm{Li}$ loss after 300 cycles. Further, as shown in Fig. 2b, Li (red), Ni (green), Co (violet), $\mathrm{Mn}$ (orange) and $\mathrm{O}$ (blue) were all very uniformly distributed in 3D without any deficiency or segregation. To estimate the homogeneity of the elemental distribution, we performed a frequency distribution analysis. The value of the Pearson coefficient $(\mu)$, an indicator of the homogeneity of a distribution, for each constituent element remained low. In addition, the frequency distributions were close to binomial distributions along the depth during cycling, as shown in Supplementary Fig. 7 for $\mathrm{Li}, \mathrm{Ni}$ and $\mathrm{O}$, which reconfirms that the distribution is quite homogeneous. The STEM-high angle annular dark-field (HAADF) image of NCM-pristine from the identical location also shows a clear layered structure (R-3m) represented by alternating bright TM interlayers and dark $\mathrm{Li}$ interlayers, both at the surface of and inside particles (Fig. 2c, d, respectively). The atomic structures of the surface of NCMpristine and the corresponding bulk structures are superimposed in the inset of Fig. 2c. Note that $\mathrm{Li}$ and $\mathrm{O}$ atoms could not be detected in the HAADF images because of their low atomic numbers, which is a limitation of the STEM-HAADF technique. The observed regions are indicated by yellow squares in
Supplementary Fig. 8. In the intensity profile along the (003) plane, the discrete regions of strong intensity represent the TM layer with a d-spacing of $0.47 \mathrm{~nm}$ (Fig. 2e), indicating that TMs rarely migrate into the Li layer.

By contrast, the STEM and APT results of NCM-300 cycles reveal distinct changes in the atomic structures and the corresponding amount of $\mathrm{Li}$ as a function of the depth. At the top surface (R0) of NCM-300 cycles, the atomic ratio determined by APT is Li:Ni:Co:Mn:O =0.03:0.81:0.16:0.03:1.63 (Fig. 3a and Table 2), exhibiting the lowest amount of Li. Below the upper $\sim 15 \mathrm{~nm}$ region, the amount of Li gradually increased with the increasing distance from the surface, which implies that this region consists mainly of the TMs and oxygen, along with the most significant Li deficiency (Fig. 3a). The STEM-HAADF image also confirms the evolution of a Li-deficient phase such as a spinel- or rock-salt-like phase induced by the substantial TM migration from octahedral $3 \mathrm{a}$ (denoted by $3 \mathrm{a}$ ) TM sites to octahedral $3 \mathrm{~b}$ (denoted by $3 \mathrm{~b}$ ) Li sites in the surface region (Fig. 3b and Supplementary Fig. 9). The HAADF intensity profile perpendicular to the (003) plane in the dotted box in Fig. 3b shows a strong intensity between the TM layers, as denoted by red arrows (Fig. 3c). These results directly demonstrate that the disordered structure has a lower capacity to accommodate Li ions than the layered structure.

At $\sim 30 \mathrm{~nm}$ from the top surface (R1), the atomic fraction of $\mathrm{Li}$ is higher than that at the top surface (R0), which shows the value of 0.37 (Fig. 3d). The depletion of Li in R1 was suppressed by the decrease in the migration of TMs into $3 \mathrm{~b}$ Li sites. Clearly, the TMs migrated to a lesser degree than they did near the top 
a
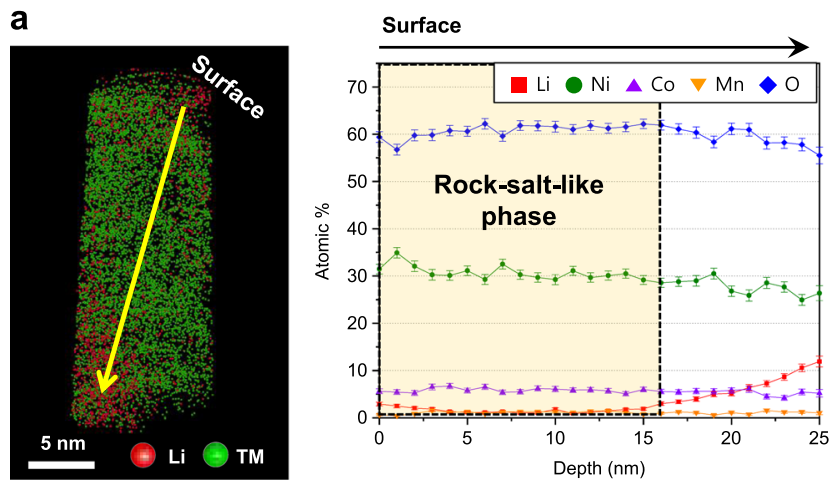

d
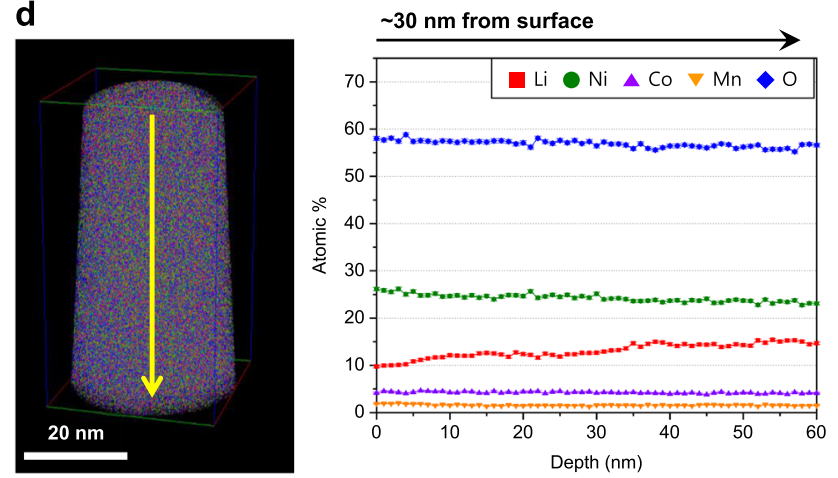

b

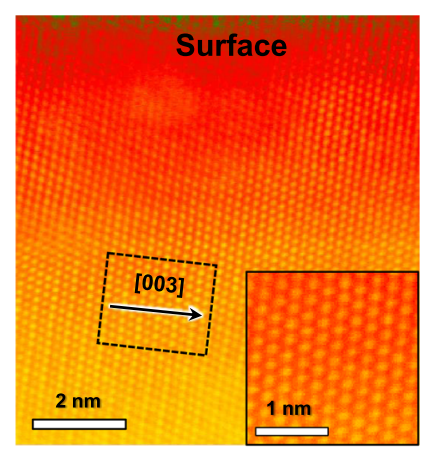

e

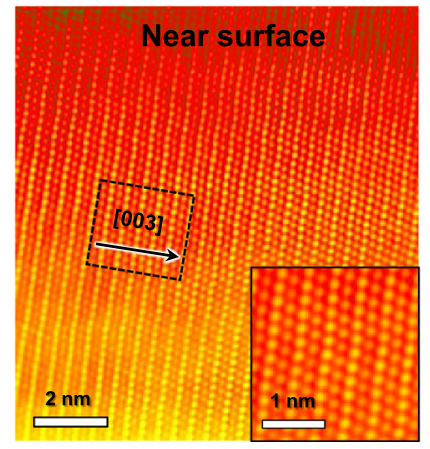

C

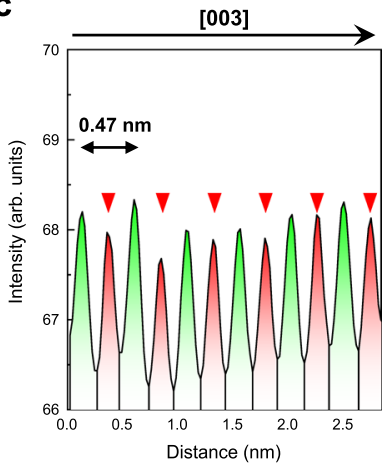

f

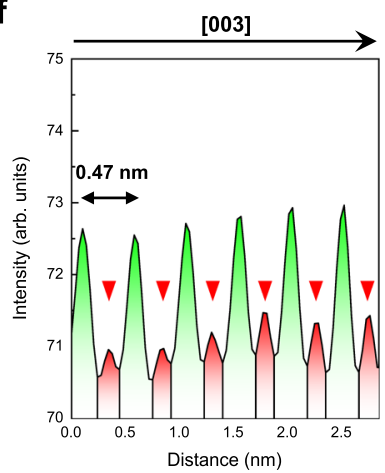

Fig. 3 Evolution of the Li deficiency and disordered structure near the surface of NCM-300 cycles. a APT depth profile at the top surface, showing a significant Li deficiency up to $15 \mathrm{~nm}$ (region enclosed by dotted square). b, c STEM-HAADF image (b) and intensity profile (c) at the top surface. The rocksalt-like phase evolves because of TM migration (inset: magnified image). Red arrows indicate TM migration from $3 b$ to $3 a$ sites. d APT depth profile starting at $\sim 30 \mathrm{~nm}$ from the top surface, showing the gradual increase in the Li concentration along the depth. e, f STEM-HAADF image (e) and intensity profile (f) starting at $~ 30 \mathrm{~nm}$ from the surface (inset: magnified image). The red arrows show that $30 \mathrm{~nm}$ below the surface, TMs migrate to a lesser extent than they do near the top surface in (c). Error bars show the standard deviation of each dataset.

surface, as denoted by red arrows (Fig. 3e, f). These stark differences in both the $\mathrm{Li}$ concentration and the structural disordering at the surface of NCM-300 cycles were mitigated in the interior of the particle. Nevertheless, at $\sim 700 \mathrm{~nm}$ from the top surface (R4), the APT depth profile still shows a slightly deficient amount of Li (Fig. 4), but this deficiency is markedly suppressed relative to that near the surface (R0-R3), showing an atomic fraction of $\mathrm{Li}$ greater than $\sim 0.70$. The partially disordered structure was also formed by just a small amount of TM migration (Fig. 4b). The HAADF intensity profile still reveals the noticeable presence of TMs in the Li layer, but the intensity is significantly weakened, as denoted by red arrows in Fig. 4c. Indeed, the central region of the particle presents almost the same amount of Li as that of NCM-pristine (Fig. 4d). Even after 300 cycles, the layered structure is well maintained in the central region of the particle, with almost no TM migration (Fig. 4e, f). Further, TM migration would not tangibly affect the atomic structure or the Li-ion distribution in the most central region. The observed regions in NCM-300 cycles are indicated by yellow squares in Supplementary Fig. 10. At low magnification, crack formation or an increased gap between primary particles was also observed after 300 cycles, as previously reported many times

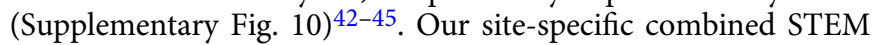
and APT analysis unequivocally confirm that the evolution of the Li concentration gradient after cycling could be attributed to structural disordering. More specifically, the capacity to accommodate $\mathrm{Li}$ ions depends on the degree of structural disordering. Therefore, the $\mathrm{Li}$ concentration gradually increases from the surface to the centre of the particle as the degree of structural disordering decreases.

\section{Discussion}

$\mathrm{Li}$ accommodation in the layered structure. Our site-specific APT results reveal the development of an increasing Li concentration gradient along the depth of the NCM particles and signify the expansion of the gradient region as cycling proceeds. Through the systematic STEM and APT observations, we further discovered that the evolution/expansion of the Li concentration gradient was associated with structural disordering. While the Li ions move toward the anode during the charging process, TMs readily move to and occupy the $3 \mathrm{~b} \mathrm{Li}$ sites in the $\mathrm{Li}$ layer via the tetrahedral sites because the formation of a disordered structure is energetically favourable in the O3-type layered structures (Fig. 5a) ${ }^{14,46-48}$. The TMs that occupy the $3 \mathrm{~b}$ Li sites are thermodynamically stable in the Li-deficient phase because of the elimination of the repulsive forces from neighbouring cationic species, which would normally facilitate TM migration. In contrast, the Li ions hardly occupy the 3a TM sites, whereas TMs occupy the $3 \mathrm{~b}$ Li sites during discharge, as shown schematically for the partially disordered structure (Fig. 5a). The inserted Li ions simply refill a few remaining vacant $3 \mathrm{~b}$ Li sites, thereby avoiding refilling the vacant $3 \mathrm{a}$ TM sites. Concurrent with the preference of TMs to migrate to $\mathrm{Li}$ sites, the prevention of $\mathrm{Li}$ migration to TM sites leads to the loss of Li accommodation sites for the inserted $\mathrm{Li}$ ions during the discharge process. The number of $\mathrm{Li}$ accommodation sites varies with the degree of structural disorder.

The particle surface in contact with the electrolyte represented as the rock-salt- or spinel-like disordered phase has a significantly lower number of $\mathrm{Li}$ accommodation sites. The interior of the particle (at a depth of $0.1-1.5 \mu \mathrm{m}$ from the 
a

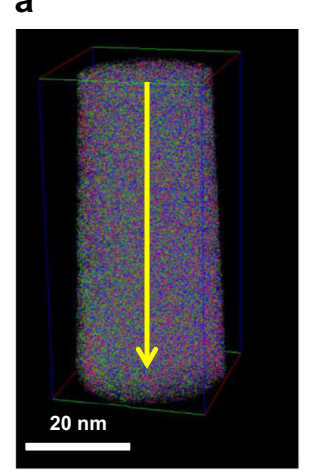

d

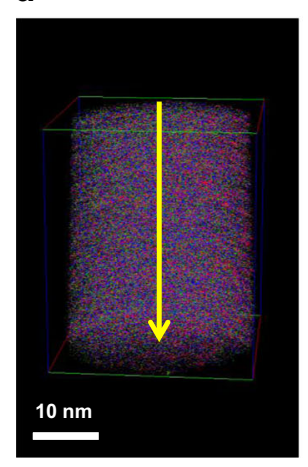

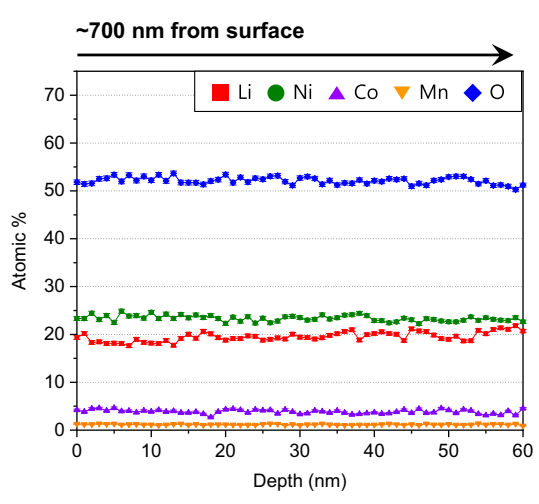

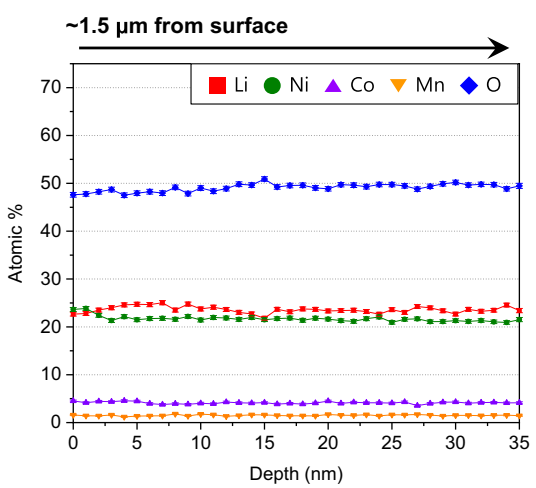

b

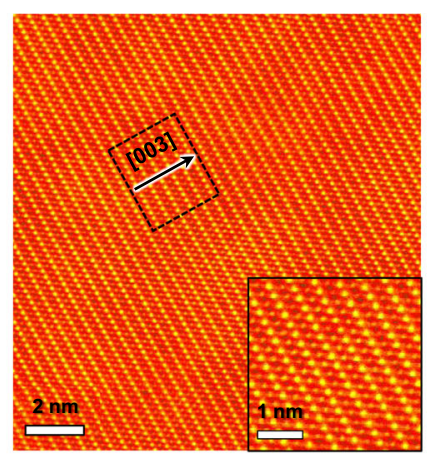

e

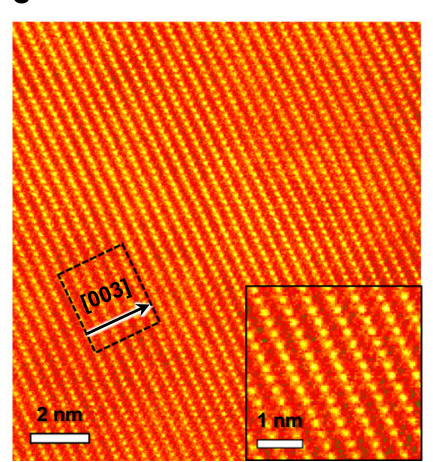

C

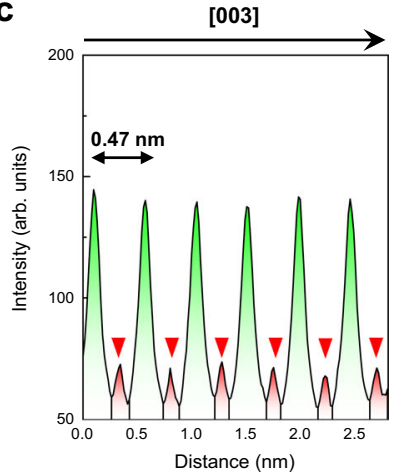

f

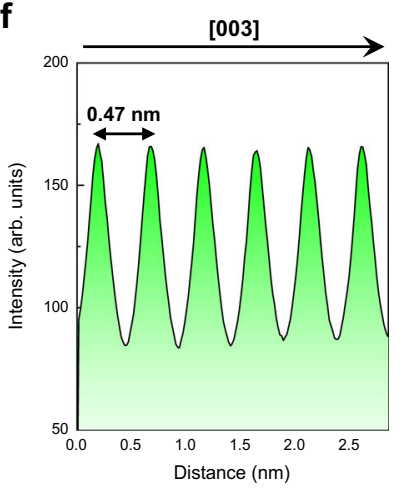

Fig. 4 Mitigation of Li deficiency and disordering in the interior of NCM-300 cycles. a APT depth profile at $700 \mathrm{~nm}$ from the top surface, showing slightly deficient Li concentration. b, c STEM-HAADF image (b) and intensity profile (c) sampled at depths of $500 \mathrm{~nm}-1 \mu \mathrm{m}$. A partially disordered structure (inset: magnified image) formed owing to the low degree of TM migration to 3a sites (red arrows) d APT depth profile in the central region of the NCM particle, showing the same Li concentration as that of NCM-pristine. e, f STEM-HAADF image (e) and intensity profile (f) in the central region. Layered structure (inset: magnified image) is well maintained, showing no TM migration. Error bars show the standard deviation of each dataset.

surface) represented by a partially disordered structure also has fewer Li accommodation sites. The centre of the particle, which retains a layered structure, has the original number of $\mathrm{Li}$ accommodation sites. Because more $\mathrm{Li}$ ions preferentially occupy the vacant Li accommodation sites, the Li concentration gradient from the surface to the centre of the particle evolves/ expands after cycling (Fig. 5b). If the inserted Li ions occupy both the vacant $\mathrm{Li}$ and TM sites, the original Li concentration should have been preserved even in the disordered structure. Hence, according to our APT and STEM results, it is reasonable that the site preference of Li ions according to the degree of structural disorder is the dominant factor that determines the total number of Li accommodation sites and consequently the Li concentration. Considering that the structural disorder correlates with the Li loss, our results provide direct evidence for the irreversible migration of TMs in the O3-type layered structures.

Non-homogeneous distribution of Li. According to our APT analysis, most of the regions in NCM show homogeneous elemental distributions with no segregation (Figs. 3 and 4 and Supplementary Fig. 11). However, we also observed the nonuniform distribution of constituent elements at several sites after cycling, in agreement with previous reports on local compositional variations ${ }^{16,33,34}$. The values of $\mu$ for $\mathrm{Li}$ are higher than 0.9 , and the observed frequency distributions significantly deviate from binomial distributions, indicating a higher degree of nonuniformity, as shown in Supplementary Fig. 12. Figure $6 a$ and $b$ shows the existence of regions locally enriched with $\mathrm{Li}$, which mainly follow a line shape. Interestingly, this line-shaped inhomogeneous Li distribution is consistent with the general shape of crystalline line defects, such as dislocations and anti-phase domain boundaries, as observed in the STEM-HAADF images (Fig. 6c, d). Although this study does not clarify the detailed relationship between the arrangement of atomic defects and $\mathrm{Li}$ accommodation sites, we could conclude that these defects change the local atomic arrangement and hence the lattice strain ${ }^{49-51}$, and eventually the distribution of $\mathrm{Li}^{51}$. The defects, which serve to concentrate strain, change the stacking sequence and stabilise the coexistence of O3- and O1-type layered structures via the formation of regions locally enriched with $\mathrm{Li}$. However, owing to the extremely low fraction of the defective volume relative to that of the entire cathode, the capacity degradation is mainly caused by the Li deficiency represented by the Li concentration gradient.

In summary, we analysed the site-specific composition of the NCM cathode material, including the Li-ion distribution, via APT. The Li concentration gradient along the radial direction in secondary particles, which decreased from a certain depth toward the particle surface, evolved after charge-discharge cycles. The depth of the gradient expanded, and the extent of Li depletion increased as the number of cycles increased, resulting in the capacity fade of LIBs. Our complementary analysis using identical-location STEM and APT reveals that the local Li deficiency originates from the lack of Li accommodation sites due to the migration of TM elements. The vacant $3 \mathrm{a}$ TM sites do not 
a

Layered structure

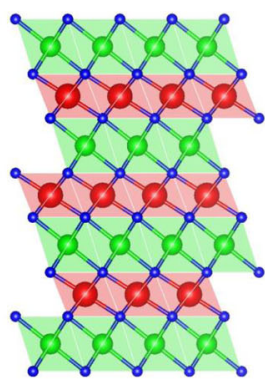

Irreversible TM migration to Li sites

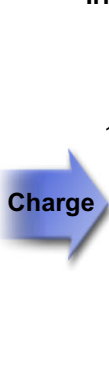

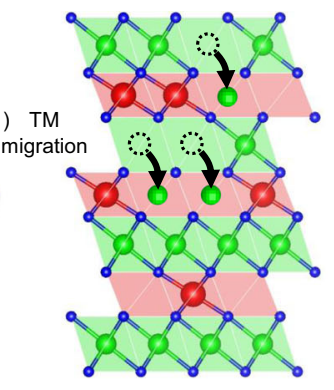

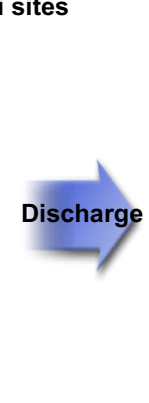

Decrease in $\mathrm{Li}$ accommodation sites and $\mathrm{Li}$ loss

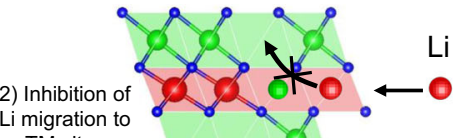

TM sites

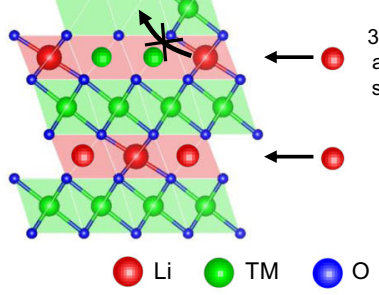

3) Decrease in $\mathrm{Li}$ accommodation sites and Li loss

b
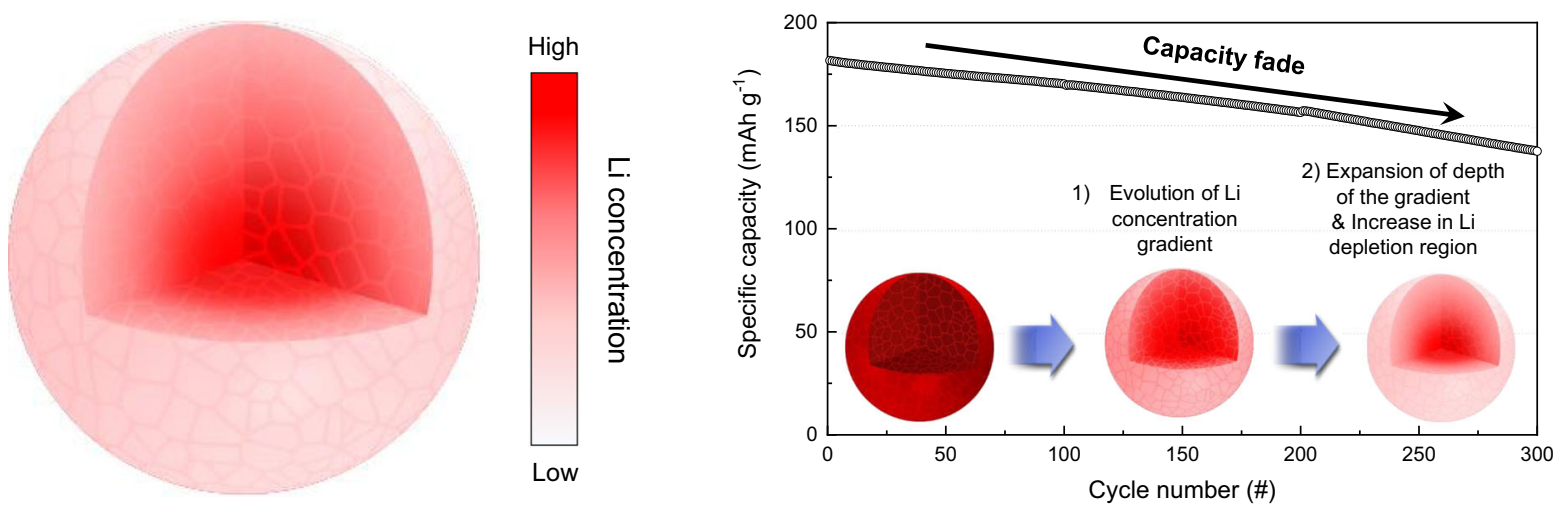

Fig. 5 Comparison of Li distribution with the increasing number of cycles. a Schematic illustration of Li accommodation sites during the charge-discharge process. TMs occupy $3 \mathrm{~b} \mathrm{Li}$ sites in the Li layer during the charging process. This irreversible TM migration depletes the number of Li accommodation sites in the Li layer. In contrast, Li ions simply refill a few vacant 3b Li sites, avoiding the 3a TM sites during the discharge process. Thus, a local Li deficiency originates from the lack of Li accommodation sites, depleted by the irreversible TM migration. $\mathbf{b}$ Schematic illustrations of the Li distribution evolution with the increasing number of cycles. The Li concentration gradient along the radial direction, which is lower at the surface and higher in the central area, evolves during cycling. The depth of the gradient and the extent of Li depletion both increase with the increasing number of cycles owing to the lack of $\mathrm{Li}$ accommodation sites.

seem to accommodate Li. Our analyses suggest that a promising strategy for improving the cycle life characteristics of LIBs is to suppress the decrease in $\mathrm{Li}$ reservoir sites during battery operation.

\section{Methods}

Synthesis of NCM. The cathode material was synthesised by a solid-state reaction. $\mathrm{LiOH} \cdot \mathrm{H}_{2} \mathrm{O}$ and co-precipitated hydroxide $\left(\left(\mathrm{Ni}_{0.80} \mathrm{Co}_{0.15} \mathrm{Mn}_{0.05}\right)(\mathrm{OH})_{2}\right)$ were used as the starting materials. To compensate for lithium loss during heat treatment, a slightly higher ratio of lithium was used with respect to the mixed metal hydroxide $(\mathrm{Li} /(\mathrm{Ni}+\mathrm{Co}+\mathrm{Mn})=1.03)$. The starting materials were mixed in a Hansel mixer at $1000 \mathrm{rpm}$ for $15 \mathrm{~min}$. Subsequently, the mixture $(150 \mathrm{~g})$ was heated at $750{ }^{\circ} \mathrm{C}$ under an air stream $(20 \mathrm{~L} / \mathrm{min})$ for $8 \mathrm{~h}$.

Electrochemical measurements. The electrochemical properties of the synthesised materials were evaluated with the use of cylindrical 18,650 cells (Supplementary Fig. 1). Artificial graphite was used as the anode. For preparing the cathode, the synthesised powder was mixed with acetylene black and a PVDF binder in 92:4:4 ratio (wt.\%). The loading level of the cathode was $17.7 \pm 0.1 \mathrm{mg} /$ $\mathrm{cm}^{2}$. A solution of $1.15 \mathrm{M} \mathrm{LiPF}_{6}$ in a mixture of ethylene carbonate/ethyl methyl carbonate/dimethyl carbonate (1:1:3 vol.\%) was used as the electrolyte. For the activation of the cells, a charge-discharge cycle of the cylindrical cell at $0.2 \mathrm{C}$ was performed twice between 4.35 and $2.8 \mathrm{~V}$ at $45^{\circ} \mathrm{C}$, and the capacity retentions were subsequently recorded at $1.0 \mathrm{C}$ and a temperature of $45^{\circ} \mathrm{C}$ in the same voltage range for 300 cycles. All cells were disassembled in a humidity-controlled room after being discharged.

STEM imaging. The cross-sectional STEM specimens were fabricated with the use of a focused ion beam (FIB, Helios Nano-Lab 600). To minimise the damage caused by the Ga-ion beam, final milling was performed using a voltage of $5 \mathrm{kV}$ and $2 \mathrm{kV}$ at currents of $16 \mathrm{pA}$ and $3 \mathrm{pA}$, respectively. The microstructure of the NCM particles was characterised by CS-corrected STEM (FEI-Titan G2 and Titan Cubed). To better visualise the structure, the region of interest is magnified and cropped (Supplementary Fig. 13).

APT specimen preparation. The needle-shaped specimens for APT were fabricated by a FIB lift-out method. On the top surface of the NCM particles, a 100-nm-thick Ag layer was deposited by sputtering at room temperature for passivation. The NCM particles were dispersed on a piece of carbon tape attached to a Si substrate. For additional passivation, a $12 \times 2 \mu \mathrm{m}$ region of interest on the particle surface was deposited with $100 \mathrm{~nm}$ thick Pt and $1-\mu \mathrm{m}-$ thick Pt layers using an electron beam and a Ga-ion beam in FIB, respectively. The passivated region of interest was lifted off and transferred onto a presharpened $\mathrm{W}$ tip. These samples were sharpened in an annular pattern using a voltage of $30 \mathrm{kV}$ at a current of $93 \mathrm{pA}$. To resharpen the samples and minimise the Ga-ion beam damage, final milling was performed using a current of 5 and $2 \mathrm{kV}$ at currents of 16 and $3 \mathrm{pA}$, respectively. To compare the composition as a function of the depth from the particle surface using APT, the distance of the analysed location from the particle surface was measured in the FIB electron beam image.

APT analysis. APT analysis was performed using a CAMECA local electrode atom probe (LEAP) $4000 \mathrm{X}$ HR in the UV laser mode $(\lambda=355 \mathrm{~nm})$ at a $125 \mathrm{kHz}$ pulse repetition rate and $40 \mathrm{~K}$ base temperature. To avoid the effect of the APT analysis conditions on the measured chemical composition of NCM, we used laser-pulsed energy of $25 \mathrm{pJ}$ at 0.005 atoms/pulse detection rate. Five APT specimens were analysed at each depth in the NCM particles to verify the concentration gradient. APT reconstruction and data processing were carried out using CAMECA Integrated Visualisation and Analysis (IVAS) software. SEM and TEM images of the tips were used for APT reconstructions. The APT mass spectrum of NCM is 
a
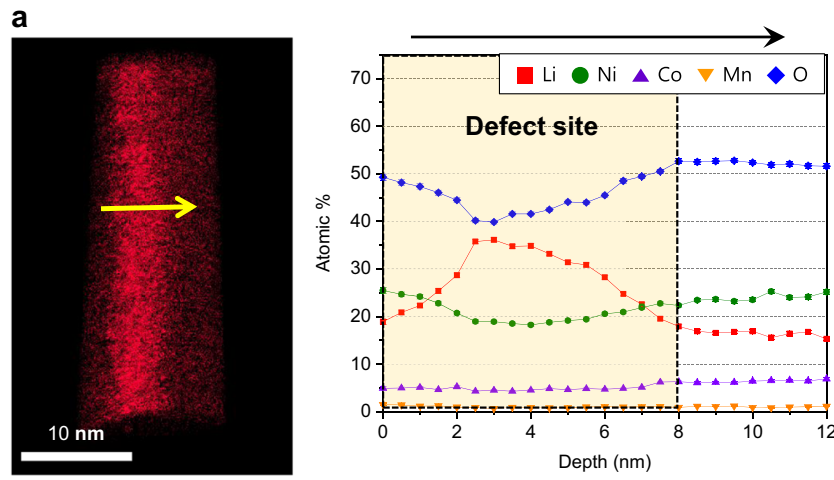

c

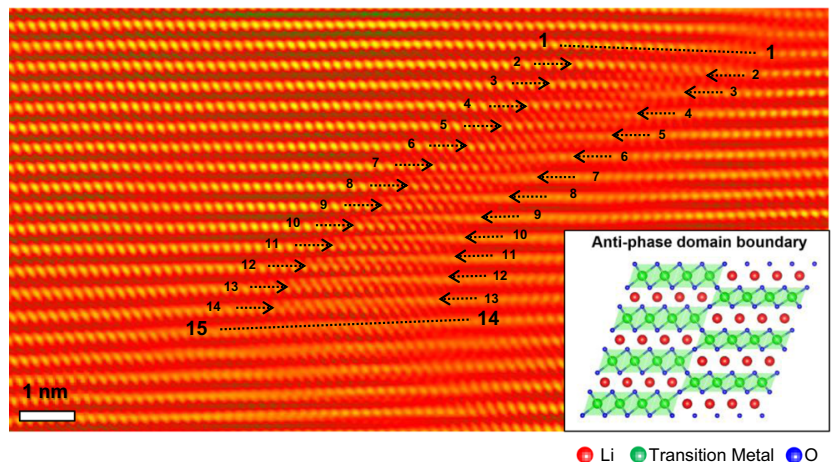

b
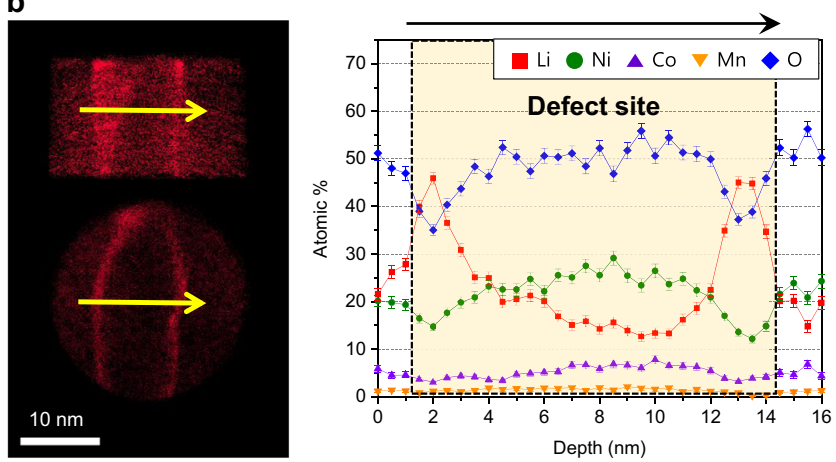

d

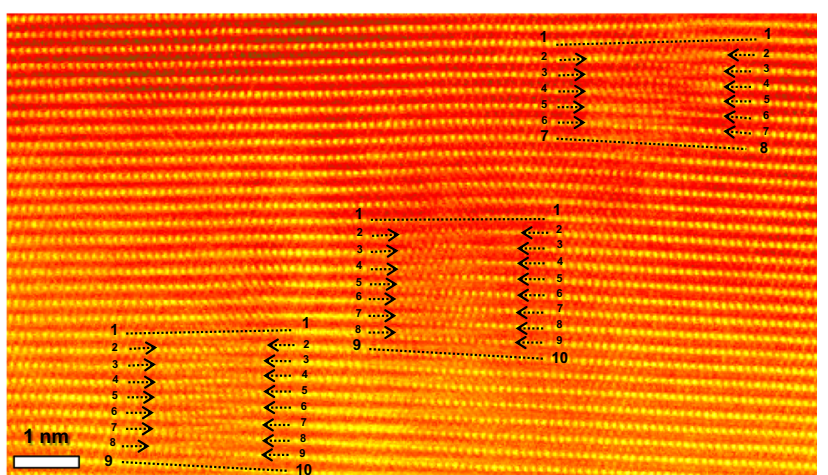

Fig. 6 Non-homogeneous distribution of $\mathbf{L i}$ and formation along line-shaped defects after charge-discharge cycles. $\mathbf{a}$, $\mathbf{b}$ APT Li map and composition profile across line-shaped Li-enriched regions. The Li concentration is locally high, following a line shape. Error bars show the standard deviation of each dataset. $\mathbf{c}, \mathbf{d}$ STEM-HAADF images of the anti-phase domain boundary (APDB) (c) and the arrangement of APDB in the grain (d). Inset in (c) is a schematic illustration of APDB.

exhibited in Supplementary Fig. 14. A pole is not visible in our APT data, as shown in Supplementary Fig. 15.

\section{Data availability}

The data that support the findings of this study are available from the corresponding author upon reasonable request.

Received: 22 December 2020; Accepted: 3 June 2021;

Published online: 21 June 2021

\section{References}

1. Dunn, B., Kamath, H. \& Tarascon, J. M. Electrical energy storage for the grid: a battery of choices. Science 334, 928-935 (2011).

2. Manthiram, A. An outlook on lithium ion battery technology. ACS Cent. Sci. 3, 1063-1069 (2017).

3. Etacheri, V., Marom, R., Elazari, R., Salitra, G. \& Aurbach, D. Challenges in the development of advanced Li-ion batteries: a review. Energy Environ. Sci. 4, 3243-3262 (2011).

4. Lu, L., Han, X., Li, J., Hua, J. \& Ouyang, M. A review on the key issues for lithium-ion battery management in electric vehicles. J. Power Sources 226, 272-288 (2013).

5. Liu, W. et al. Nickel-rich layered lithium transition-metal oxide for high-energy lithium-ion batteries. Angew. Chem. Int. Ed. 54, 4440-4457 (2015).

6. Goodenough, J. B. \& Park, K. S. The Li-ion rechargeable battery: a perspective. J. Am. Chem. Soc. 135, 1167-1176 (2013).

7. Larcher, D. \& Tarascon, J. M. Towards greener and more sustainable batteries for electrical energy storage. Nat. Chem. 7, 19-29 (2014).

8. Schipper, F. et al. Recent advances and remaining challenges for lithium ion battery cathodes I. Nickel-rich, $\mathrm{LiNi}_{\mathrm{x}} \mathrm{Co}_{\mathrm{y}} \mathrm{Mn}_{\mathrm{z}} \mathrm{O}_{2}$. J. Electrochem. Soc. 164, A6220-A6228 (2017).

9. Kim, N. Y., Yim, T., Song, J. H., Yu, J. S. \& Lee, Z. Microstructural study on degradation mechanism of layered $\mathrm{LiNi}_{0.6} \mathrm{Co}_{0.2} \mathrm{Mn}_{0.2} \mathrm{O}_{2}$ cathode materials by analytical transmission electron microscopy. J. Power Sources 307, 641-648 (2016).

10. Jung, S. K. et al. Understanding the degradation mechanisms of $\mathrm{LiNi}_{0.5} \mathrm{Co}_{0.2} \mathrm{Mn}_{0.3} \mathrm{O}_{2}$ cathode material in lithium ion batteries. Adv. Energy Mater. 4, 1300787 (2014).

11. Yan, P. et al. Evolution of lattice structure and chemical composition of the surface reconstruction layer in $\mathrm{Li}_{1.2} \mathrm{Ni}_{0.2} \mathrm{Mn}_{0.6} \mathrm{O}_{2}$ cathode material for lithium ion batteries. Nano Lett. 15, 514-522 (2014).

12. Kleiner, K. et al. Origin of high capacity and poor cycling stability of Li-rich layered oxides: a long-duration in situ synchrotron powder diffraction study. Chem. Mater. 30, 3656-3667 (2018).

13. Gu, M. et al. Formation of the spinel phase in the layered composite cathode used in Li-ion batteries. ACS Nano 7, 760-767 (2012).

14. Eum, D. et al. Voltage decay and redox asymmetry mitigation by reversible cation migration in lithium-rich layered oxide electrodes. Nat. Mater. 19, 419-427 (2020)

15. Feng, X. et al. Performance improvement of Li-rich layer-structured $\mathrm{Li}_{1.2} \mathrm{Mn}_{0.54} \mathrm{Ni}_{0.13} \mathrm{Co}_{0.13} \mathrm{O}_{2}$ by integration with spinel $\mathrm{LiNi}_{0.5} \mathrm{Mn}_{1.5} \mathrm{O}_{4}$. Phys. Chem. Chem. Phys. 17, 1257-1264 (2015).

16. Devaraj, A. et al. Visualizing nanoscale 3D compositional fluctuation of lithium in advanced lithium-ion battery cathodes. Nat. Commun. 6, 8014 (2015).

17. Pearce, P. E. et al. Evidence for anionic redox activity in a tridimensionalordered Li-rich positive electrode $\beta-\mathrm{Li}_{2} \mathrm{IrO}_{3}$. Nat. Mater. 16, 580 (2017).

18. Lee, S. Y. et al. Revisiting primary particles in layered lithium transition-metal oxides and their impact on structural degradation. Adv. Sci. 6, 1800843 (2019).

19. Zhou, Y. N. et al. Tuning charge-discharge induced unit cell breathing in layer-structured cathode materials for lithium-ion batteries. Nat. Commun. 5, 5381 (2014).

20. Wen, Y., Shang, T. \& Gu, L. Analytical ABF-STEM imaging of Li ions in rechargeable batteries. Microscopy 66, 25-38 (2017).

21. Wang, Z. et al. In situ STEM-EELS observation of nanoscale interfacial phenomena in all-solid-state batteries. Nano Lett. 16, 3760-3767 (2016).

22. Akita, T. \& Taguchi, N. Practical analysis of Li distribution by EELS. Sur. Interface Anal. 48, 1226-1230 (2016).

23. Gao, $\mathrm{X}$. et al. Oxygen loss and surface degradation during electrochemical cycling of lithium-ion battery cathode material $\mathrm{LiMn}_{2} \mathrm{O}_{4}$. J. Mater. Chem. A 7 , 8845-8854 (2019). 
24. $\mathrm{Gu}, \mathrm{M}$. et al. Nanoscale phase separation, cation ordering, and surface chemistry in pristine $\mathrm{Li}_{1.2} \mathrm{Ni}_{0.2} \mathrm{Mn}_{0.6} \mathrm{O}_{2}$ for Li-ion batteries. Chem. Mater. 25, 2319-2326 (2013).

25. Yang, S. et al. Soft X-ray XANES studies of various phases related to $\mathrm{LiFePO}_{4}$ based cathode materials. Energy Environ. Sci. 5, 7007-7016 (2012).

26. Weker, J. N. et al. In situ nanotomography and operando transmission X-ray microscopy of micron-sized Ge particles. Energy Environ. Sci. 7, 2771-2777 (2014).

27. Yang, F. et al. Nanoscale morphological and chemical changes of high voltage lithium-manganese rich NMC composite cathodes with cycling. Nano Lett. 14, 4334-4341 (2014).

28. Pongha, S. et al. XANES investigation of dynamic phase transition in olivine cathode for Li-ion batteries. Adv. Energy Mater. 5, 1500663 (2015).

29. Wang, J., Eng, C., Chen-Wiegart, Y. C. K. \& Wang, J. Probing threedimensional sodiation-desodiation equilibrium in sodium-ion batteries by in situ hard X-ray nanotomography. Nat. Commun. 6, 7496 (2015).

30. Wang, J., Chen-Wiegart, Y. C. K., Eng, C., Shen, Q. \& Wang, J. Visualization of anisotropic-isotropic phase transformation dynamics in battery electrode particles. Nat. Commun. 7, 12372 (2016)

31. Kelly, T. F. \& Larson, D. J. Atom probe tomography 2012. Annu. Rev. Mater. Sci. 42, 1-31 (2012).

32. Gault, B., Moody, M. P., Cairney, J. M. \& Ringer, S. P. in Atom Probe Microscopy, Springer Series in Material Science (eds Hull, R. \& Jagadish, C.) Vol. 160, 28-64 (Springer, 2012).

33. Diercks, D. R. et al. Evidence for anisotropic mechanical behavior and nanoscale chemical heterogeneity in cycled $\mathrm{LiCoO}_{2}$. J. Electrochem. Soc. 161, F3039-F3045 (2014)

34. Maier, J., Pfeiffer, B., Volkert, C. A. \& Nowak, C. Three-dimensional microstructural characterization of lithium manganese oxide with atom probe tomography. Energy Technol. 4, 1565-1574 (2016).

35. Myung, S. T. et al. Nickel-rich layered cathode materials for automotive lithium-ion batteries: achievements and perspectives. ACS Energy Lett. 2 196-223 (2016).

36. Devaraj, A., Colby, R., Hess, W. P., Perea, D. E. \& Thevuthasan, S. Role of photoexcitation and field ionization in the measurement of accurate oxide stoichiometry by laser-assisted atom probe tomography. J. Phys. Chem. Lett. 4, 993-998 (2013).

37. Silaeva, E. P., Karahka, M. \& Kreuzer, H. J. Atom probe tomography and field evaporation of insulators and semiconductors: Theoretical issues. Curr. Opin. Solid State Mater. Sci. 17, 211-216 (2013).

38. Gault, B. et al. Behavior of molecules and molecular ions near a field emitter. N. J. Phys. 18, 033031 (2016).

39. Zanuttini, D. et al. Simulation of field-induced molecular dissociation in atomprobe tomography: Identification of a neutral emission channel. Phys. Rev. A 95, 061401 (2017).

40. Meisenkothen, F. et al. Effects of detector dead-time on quantitative analyses involving boron and multi-hit detection events in atom probe tomography. Ultramicroscopy 159, 101-111 (2015)

41. Peng, Z. et al. On the detection of multiple events in atom probe tomography. Ultramicroscopy 189, 54-60 (2018).

42. Sun, H. H. \& Manthiram, A. Impact of microcrack generation and surface degradation on a nickel-rich layered $\mathrm{Li}\left[\mathrm{Ni}_{0 .}{ }_{9} \mathrm{Co}_{0 .}{ }_{05} \mathrm{Mn}_{0 .}{ }_{05}\right] \mathrm{O}_{2}$ cathode for lithium-ion batteries. Chem. Mater. 29, 8486-8493 (2017).

43. Park, K. J. et al. Degradation mechanism of Ni-enriched NCA cathode for lithium batteries: are microcracks really critical? ACS Energy Lett. 4, 1394-1400 (2019).

44. Kondrakov, A. O. et al. Anisotropic lattice strain and mechanical degradation of high-and low-nickel NCM cathode materials for Li-ion batteries. J. Phys. Chem. C. 121, 3286-3294 (2017)

45. Park, S. Y. et al. Probing electrical degradation of cathode materials for lithium-ion batteries with nanoscale resolution. Nano Energy 49, 1-6 (2018).
46. Lim, J. M. et al. The origins and mechanism of phase transformation in bulk $\mathrm{Li}_{2} \mathrm{MnO}_{3}$ : first-principles calculations and experimental studies. J. Mater. Chem. A 3, 7066-7076 (2015).

47. Lee, E. \& Persson, K. A. Structural and chemical evolution of the layered Liexcess $\mathrm{Li}_{\mathrm{x}} \mathrm{MnO}_{3}$ as a function of Li content from first-principles calculations. Adv. Energy Mater. 4, 1400498 (2014).

48. Reed, J. \& Ceder, G. Role of electronic structure in the susceptibility of metastable transition-metal oxide structures to transformation. Chem. Rev. 104, 4513-4534 (2004).

49. Li, Q. et al. Dynamic imaging of crystalline defects in lithium-manganese oxide electrodes during electrochemical activation to high voltage. Nat. Commun. 10, 1692 (2019).

50. Wang, R. et al. Atomic structure of $\mathrm{Li}_{2} \mathrm{MnO}_{3}$ after partial delithiation and relithiation. Adv. Energy Mater. 3, 1358-1367 (2013).

51. Singer, A. et al. Nucleation of dislocations and their dynamics in layered oxide cathode materials during battery charging. Nat. Energy 3, 641-647 (2018).

\section{Acknowledgements}

We thank Seongwoo Hwang, the former corporate president of SAIT, for helpful discussions.

\section{Author contributions}

E.L., W.S.J., and S.Y.P. conceived the research plan. J.H.S. synthesised Li

$\left(\mathrm{Ni}_{0.80} \mathrm{Co}_{0.15} \mathrm{Mn}_{0.05}\right) \mathrm{O}_{2}$ and tested cells. B.G.C. conducted the APT study. S.Y.P. and B.G. C. conducted TEM. B.G.C. and S.Y.P. wrote the paper. All authors discussed the results and commented on the manuscript.

\section{Competing interests}

The authors declare no competing interests.

\section{Additional information}

Supplementary information The online version contains supplementary material available at https://doi.org/10.1038/s41467-021-24120-w.

Correspondence and requests for materials should be addressed to B.-G.C. or S.Y.P.

Peer review information Nature Communications thanks Baptiste Gault, Jonathan Poplawsky and the other, anonymous, reviewer(s) for their contribution to the peer review of this work. Peer reviewer reports are available.

Reprints and permission information is available at http://www.nature.com/reprints

Publisher's note Springer Nature remains neutral with regard to jurisdictional claims in published maps and institutional affiliations.

Open Access This article is licensed under a Creative Commons Attribution 4.0 International License, which permits use, sharing, adaptation, distribution and reproduction in any medium or format, as long as you give appropriate credit to the original author(s) and the source, provide a link to the Creative Commons license, and indicate if changes were made. The images or other third party material in this article are included in the article's Creative Commons license, unless indicated otherwise in a credit line to the material. If material is not included in the article's Creative Commons license and your intended use is not permitted by statutory regulation or exceeds the permitted use, you will need to obtain permission directly from the copyright holder. To view a copy of this license, visit http://creativecommons.org/ licenses/by/4.0\%

(c) The Author(s) 2021 